\title{
Spectral Model for Fixed-Pattern-Noise in Infrared Focal-Plane Arrays ${ }^{\star}$
}

\author{
Jorge E. Pezoa and Osvaldo J. Medina \\ Departamento de Ingeniería Eléctrica and Center for Optics and Photonics (CEFOP) \\ Universidad de Concepción, Concepción, Chile \\ \{jpezoa, osvaldomedina\}@udec.cl \\ http://www.telecomunicaciones.udec.cl
}

\begin{abstract}
In this paper a novel and more realistic analytical model for the fixed-pattern noise present in infrared focal plane arrays is developed. The model captures, in the frequency domain, the spatial structure of the fixed-pattern noise yielding a suitable input/output representation for an infrared focal plane array. The theoretical and practical applicability the model is illustrated by both synthesizing fixed-pattern noise from three different infrared cameras and improving the performance of a previously reported fixed-pattern noise compensation algorithm.
\end{abstract}

\section{Introduction}

Infrared cameras are being used in a wide range of applications such as temperature measurement, spectral-signature analysis, night vision, and predictive machinery maintenance. Infrared cameras collect the spectral information by means of imaging sensing devices called infrared focal plane arrays (FPAs). An infrared FPA is an integrated circuit composed of either a linear or a matrix of infrared photodetectors. An image is created after reading and properly assembling the infrared information collected by all the photodetectors in the array [1]. In theory, all the photodetectors in the FPA respond in exactly the same manner when a spatially uniform spectral object is placed in front of the array. Unfortunately practical FPA do not respond in such a way when a flat infrared input impinges the array.

The fixed-pattern noise (FPN) in infrared FPAs is the nonuniform spatial response of the FPA when a spatially uniform stimulus is used as an input. The FPN is a quasy-stationary, spatially structured type of noise, which produces a grid-like pattern on top of the true images. The FPN is attributed to device manufacturing mismatches and parameter variations across the FPA, such as photodetector area and dark current [2,3]. Since the grid-like pattern severely degrades the true images, it is mandatory to compensate for the FPN before using the imagery acquired by an infrared camera equipped with an FPA.

Surprisingly, the spatial structure of the FPN is oversimplified in the literature under the assumption that the FPN is spatially independent [4,5, 6. Only few

\footnotetext{
* Authors acknowledge the support of CEFOP and Grant CONICYT PFB-0824.

C. San Martin and S.-W. Kim (Eds.): CIARP 2011, LNCS 7042, pp. 55-63, 2011.

(C) Springer-Verlag Berlin Heidelberg 2011
} 
papers have considered the existence of spatial structure in the FPN [7,8]; however, in none of these works specific models for the FPN have been developed. To the best of our knowledge, the work [9] by El Gamal et al. is the only paper available where a model for the FPN has been developed. Such a model was developed in the spatial domain by means of random fields and was specifically developed for modeling noise in CMOS sensors.

In this work we have tackled the necessity of supplying appropriate theoretical models for FPN. To do so, we have developed a novel analytical model for the spatial structure of FPN. The model has been developed in the frequency domain after experimentally characterizing the spectrum of several infrared FPAs. The analytic characterization models the magnitude of the FPN using a linear combination of unidimensional second-order exponential functions, while the phase of the FPN is assumed to be random and uniformly distributed. The analytic model is completed by deriving a total least-squares estimator for the FPN parameters. The main advantage of the model presented here is that it yields a more realistic input/output representation for an entire infrared FPA. The theoretical and practical applicability of our model is illustrated by both synthesizing FPN from three different IR cameras and improving the performance of a previously reported FPN compensation algorithm.

The rest of this paper is organized as follows. Section 2 presents the spectral analysis of FPN conducted using experimental data. In the same section, an analytical model for the FPN in the frequency domain is developed using simple mathematical expressions with a small number of parameters. The ability of the analytical model developed in Section 2.2 to represent the FPN is verified in Section 3 by means of simulations and experimental data. Finally, in Section 4 the conclusions of our work are outlined.

\section{Analytical Model of FPN}

\subsection{Spectral Analysis}

Theoretically speaking, the spatial output of an ideal infrared FPA to a flat input should be flat; in the frequency domain, such ideal response should be observed as a delta function. However, when an actual infrared FPA is illuminated by a blackbody source, the observed output is not flat and in the spatial frequency domain a specific shape is observed. By assuming that the FPN has only an additive component, [10, 4, 5, 6], the shape observed in the frequency domain is precisely the frequency-domain representation of the FPN. To obtain a realistic model, an experimental spectral analysis of the FPN has been conducted by collecting data from different infrared cameras illuminated with black-body radiator sources.

For instance, Fig. 1(a) shows an actual sample-frame taken with a CEDIP brand infrared camera. The size of the frame is $240 \times 320$ pixels and a black-body source at a temperature of $20^{\circ} \mathrm{C}$ was employed as an input. Note that, a grid-like pattern is observed instead of a flat image. Note also that, from basic Fourier theory, a grid-like FPN should be represented in the Fourier domain by a crosslike shape. Figure1(b) shows the magnitude of the fast Fourier transform (FFT) 
of the sample frame shown in Fig.11(a). In this figure the magnitude of the Fourier transform of both the flat input and the FPN can be observed. In the Fourier domain, the flat input corresponds to the impulse at the center of the spectrum, while the FPN has a clearly defined structure with one vertical spectral band and one horizontal spectral band. Regarding the phase representation of the FPN, in our analysis we have observed a lack of structure in the phase. Such behavior can be justified by the isotropic behavior of the FPN.

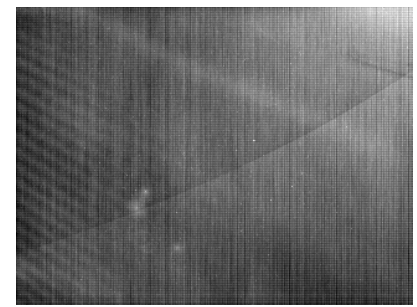

(a)

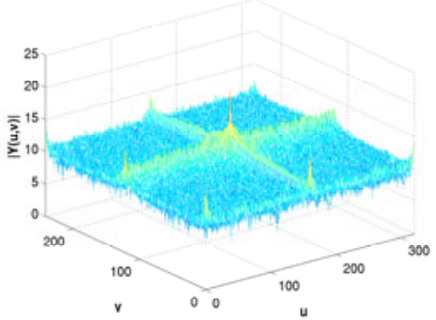

(b)

Fig. 1. (a) Sample frame of FPN. (b) Magnitude of the FFT of the sample frame.

Finally, our experience a grid-like pattern is a very common type of FPN in practical devices. Consequently, for the remaining of this paper we will assume that the FPN is in fact represented, in the Fourier domain, by one vertical and one horizontal band as those shown in Fig. 1(b). With this, we can introduce a precise analytical model for the class of all infrared sensors whose FPN manifests itself as a pattern of vertical and horizontal lines.

\section{$2.2 \quad$ Spectral Analytical Model}

From the analysis presented in the previous subsection we state that the magnitude of the FPN can be modeled as a lineal combination of two independent second-order exponential functions, each one of them having three parameters: the location parameter, the amplitude parameter, and the scale parameter. Regarding the phase, here we have assumed that it can be modeled as a white noise process, with uniform distribution in $[-\pi, \pi]$. Thus, given an FPA of size $2 M+1 \times 2 N+1$ pixels, the magnitude and phase of the FPN can be mathematically modeled as:

$$
\begin{aligned}
& |Y(u, v)|=B_{u} \exp \left(\frac{-\left(u-u_{0}\right)^{2}}{2 \sigma_{u}^{2}}\right)+B_{v} \exp \left(\frac{-\left(v-v_{0}\right)^{2}}{2 \sigma_{v}^{2}}\right), \\
& \angle Y(u, v) \sim U[-\pi, \pi]
\end{aligned}
$$

where $u=-M, \ldots, M, v=-N, \ldots, N$, and $Y(u, v)$ is the two-dimensional Fourier transform of the FPN. The parameters $u_{0}, B_{u}$, and $\sigma_{u}$ (correspondingly, $v_{0}, B_{v}$, and $\sigma_{v}$ ) are the location, the amplitude, and the scale, respectively, of the horizontal (correspondingly, vertical) band. 


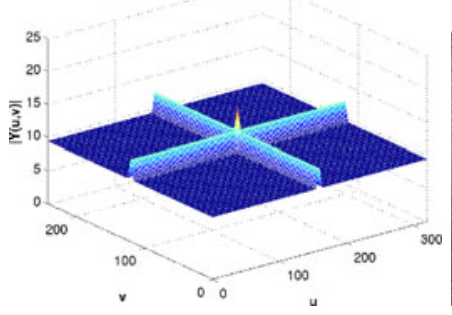

(a)

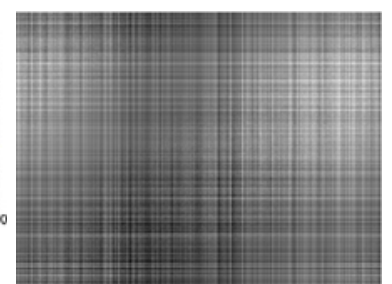

(b)

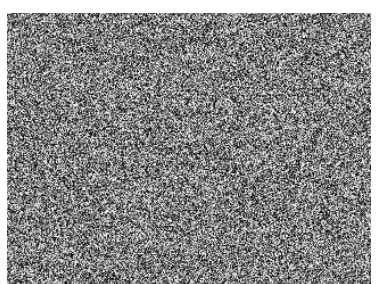

(c)

Fig. 2. Synthetic versions of the FPN shown in Fig. 11 (a) Magnitude spectrum, and (b) Spatially structured. (c) Sample frame of spatially unstructured fixed-pattern noise.

For completeness, we have developed also in this paper parameter estimators for (1). We provide only formulae for estimating $u_{0}, B_{u}$, and $\sigma_{u}$. Estimators for $v_{0}, B_{v}$, and $\sigma_{v}$ can be obtained mutatis mutandis. First, note that the location parameter for the second-order exponential functions must be zero due to the geometry of the FPN, i.e. $u_{0}=0$. Next, by exploiting the total least-squares error principle and parameter estimators for $B_{u}$ and $\sigma_{u}$ are given by the following non-linear system of equations:

$$
\begin{aligned}
(2 M+1) \ln \hat{B}_{u}-\sum_{u=-M}^{M}\left(\ln (Y(u))+\left(2 \hat{\sigma}_{u}^{2}\right)^{-1}\left(u-u_{0}\right)^{2}\right) & =0, \\
\sum_{u=-M}^{M}\left(u-u_{0}\right)^{4}-2 \hat{\sigma}_{u}^{2} \sum_{u=-M}^{M}\left(u-u_{0}\right)^{2}\left(\ln \hat{B}_{u}-\ln (Y(u))\right) & =0 .
\end{aligned}
$$

\section{Results}

The ability of the analytical model to represent the FPN is verified in this section. We have synthesized FPN using the traditional approach taken in the literature, that is, samples of FPN are drawn from a white spatial process following an uniform distribution supported on the same dynamical range as the raw data. Such samples of unstructured FPN are compared to synthetic, spatially structured FPN generated using the model (11) and (2).

First, we aim to mimic the actual FPN of the CEDIP camera shown in Fig. 1. To do so, we use (3) and (44) and estimate that $B_{u} \approx \hat{B}_{u}=5.2$ and $\sigma_{u} \approx \hat{\sigma}_{u}=2.5$, and employed these values to plot the magnitude spectrum shown in Fig. 2(a). Next, we used (11) and (2) to render samples of synthetic FPN. One of such samples is shown in Fig. 2(b). A naked-eye inspection to Figs. 2(b) and (c) shows that: (i) the synthetic FPN resembles the actual FPN shown in Fig. 1(a); and (ii) the unstructured (spatially white) noise in Fig. 2(c) is clearly not a valid representation of the FPN.

Second, we objectively compare samples of actual FPN to both unstructured synthetic FPN and synthetic FPN generated using our model. The comparison has been made by means of the root mean-squared error (RMSE) between actual and synthetic samples of FPN. Figures 3(a) to (c) show samples of actual FPN from three infrared cameras. Note that in these cameras the FPN is exhibited as a horizontal and/or vertical regular pattern. Table11ists the RMSE in synthesizing 
FPN in both the spatial and the frequency domain. These results clearly indicate that the RMSE for the structured synthetic FPN is always lower than the RMSE of the unstructured synthetic FPN. In Figs. 3(d) to (f) the magnitude spectrum of the FPN shown in Figs. 3(a) to (c) have been plotted, while in Figs. 3)(g) to (h) samples of synthetic FPN, drawn the proposed model, are shown. Clearly, the spatial structure of the actual FPN can be observed in the synthetic versions of the noise.

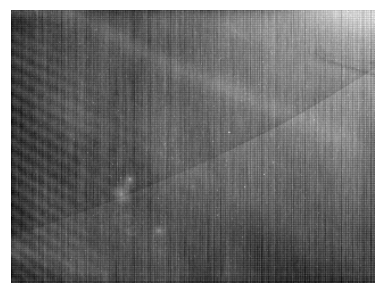

(a)

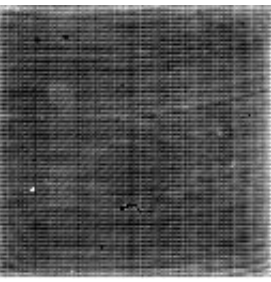

(b)

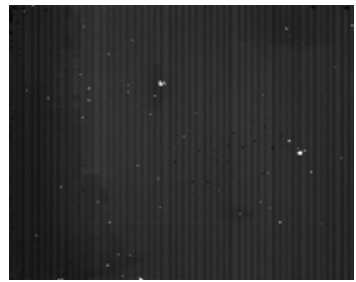

(c)

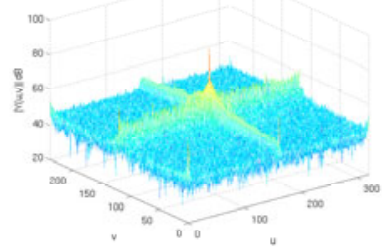

(d)

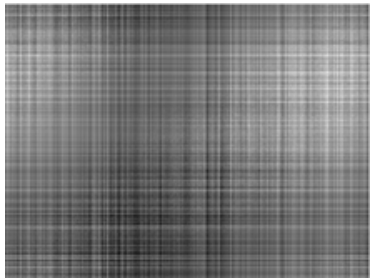

(g)

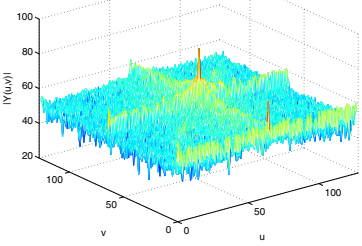

(e)

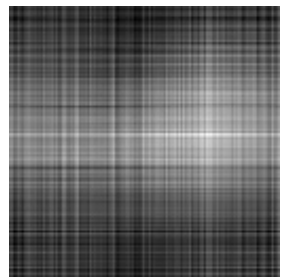

(h)

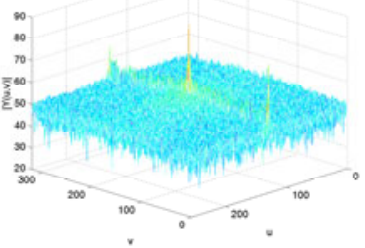

(f)

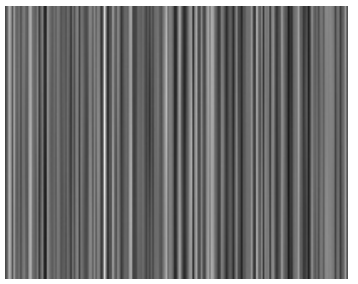

(i)

Fig. 3. FPN from different infrared cameras: (a) CEDIP. (b) Amber. (c) Quantum Dots. Magnitude spectrum of FPN: (d) CEDIP. (e) Amber. (f) Quantum Dots. A sample of ynthetic FPN: (g) CEDIP. (h) Amber. (i) Quantum Dots.

Third, in hyperspectral push-broom cameras (where a scanning procedure must be employed to create infrared images) the FPN observed as a striping pattern 11,12,13. A sample of striping FPN in hyperspectral push-broom cameras is shown in Fig. 4(a) and its magnitude spectrum is shown in Fig. 4(b). We note that the striping noise can be considered a special case of the model for the gridlike FPN we have introduced here. In particular, the mathematical expression for the magnitude of the striping noise reduces to: $|Y(u, v)|=B_{u} \exp \left(\frac{-\left(u-u_{0}\right)^{2}}{2 \sigma_{u}^{2}}\right)$. 


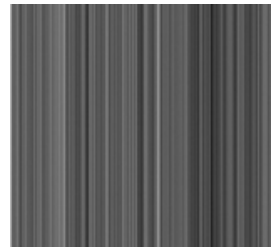

(a)

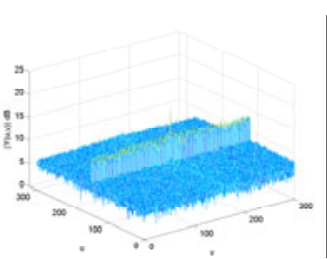

(b)

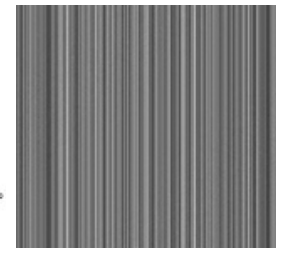

(c)

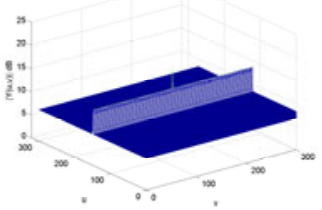

(d)

Fig. 4. (a) Actual sample of striping noise; and its (b) Magnitude spectrum. (c) Synthetic sample of striping noise; and its (d) Magnitude spectrum.

Figures 4(c) and (d) show, respectively, a sample of synthetic striping noise in the spatial domain and the magnitude spectrum of synthetic FPN.

Fourth, as a final application of our model, we have supplemented the noisecancellation-based non-uniformity correction (NUC) algorithm developed by Godoy et al. in [10] with our analytical model, thereby obtaining an enhanced version of such NUC algorithm. In [10, Godoy et al. employed spatially white FPN as the required source of correlated noise which is essential to any noisecancellation system. Here, we have replaced such noise source by our model of spatially structured FPN. Intuitively speaking, if the noise source is replaced by a proper FPN model, the performance of the NUC algorithm should be improved.

Figures [5(a) and (d) correspond to raw frames acquired using the Amber infrared camera. Figures 5(b) and (e) correspond to corrected versions of the raw frames obtained when the unstructured FPN is used as the source of correlated noise in the noise-cancellation-based NUC method, while Figs. 5(c) and (f) correspond to corrected versions of the raw frames obtained when the structured FPN is employed as the source of correlated noise. It can be observed from the figures that corrected frames obtained using the spatially structured FPN look better than those obtained using the unstructured FPN. This result is important because it confirms the intuition that, only by substituting the source of FPN the performance of the noise-cancellation-based NUC algorithm can be improved.

In addition to the subjective evaluation, a quantitative performance analysis between the original and the enhanced versions of the NUC algorithm was conducted by means of the so-called roughness metric. The roughness metric is employed to assess NUC methods when no reference images are available. The metric is a combination of high-pass spatial filters aiming to measure the sharp changes in

Table 1. RMSE in synthesizing FPN for different cameras

\begin{tabular}{l|c|c|c|c}
\hline \hline & \multicolumn{2}{|c|}{ Spatial domain } & \multicolumn{2}{c}{ Spectral domain } \\
& Structured & Unstructured & Structured & Unstructured \\
\hline CEDIP & 0.3180 & 0.4248 & 8.6782 & 19.8853 \\
Amber & 0.1378 & 0.3028 & 11.0094 & 23.5877 \\
Quantum Dots & 0.3746 & 0.4485 & 11.3907 & 22.9642 \\
\hline \hline
\end{tabular}




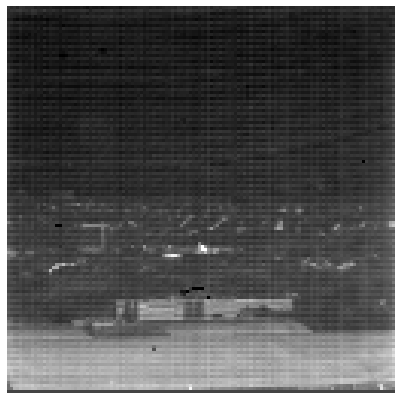

(a)

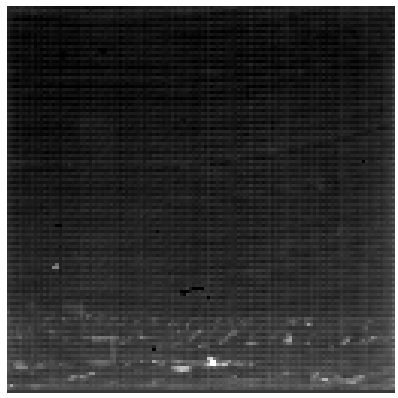

(d)

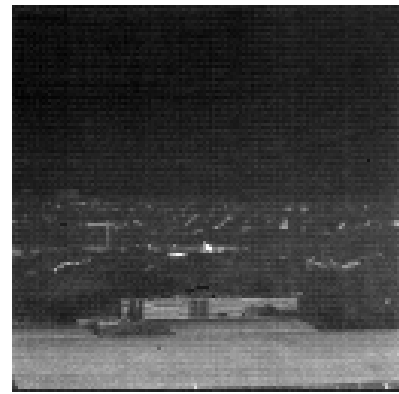

(b)

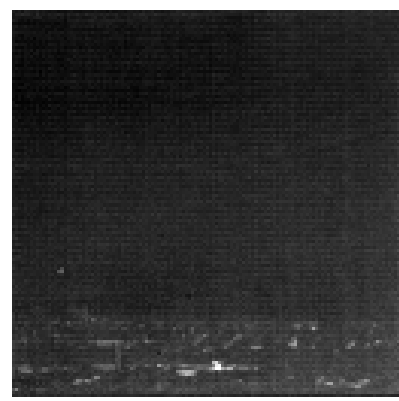

(e)

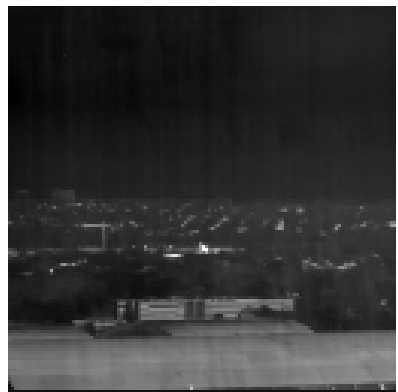

(c)

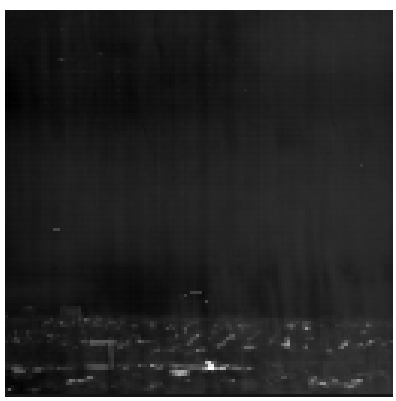

(f)

Fig. 5. (a) and (d) Sample frames corrupted by FPN. (b) and (e) Corrected versions of previous frames when unstructured FPN is employed. (c) Corrected versions of previous frames when spatially structured FPN is employed.

both the horizontal and the vertical directions. By definition, the roughness metric achieves only non-negative values, a better the performance is mapped onto values for roughness closer to zero. (The mathematical definition of the metric can be

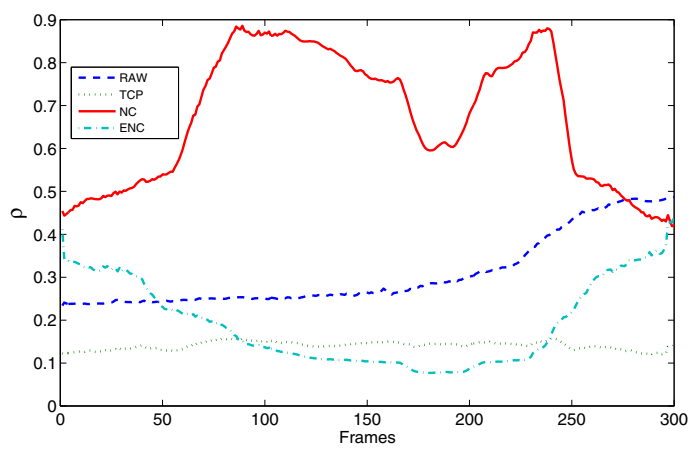

Fig. 6. Roughness comparison. RAW: Noisy frame. TPC: Two-point calibration. NC: Noise-cancellation algorithm. ENC: Noise-cancellation algorithm with structured FPN. 
found elsewhere [10].) Figure 6 shows the results of the assessment. For comparison in Fig. 6] we have plotted also the worst-case scenario, labeled as RAW, corresponding to the roughness metric of the noisy images, and the best-case scenario, labeled as TPC, corresponding to a laboratory calibration [2,10]. In Fig. 6] can be observed that the FPN compensation using spatially structured noise, labeled as ENC, is indeed better than the correction obtained using unstructured FPN, labeled as NC. The improved performance is attributed to the spatially structured noise source supplied by our model.

\section{Conclusions}

In this work, the spatial structure of the FPN corrupting infrared FPAs has been analytically modeled in the frequency domain. Our model represents the FPN in the frequency domain by means of two components: a deterministic part, which defines the spatial structure, and a random part, which characterizes the phase. Our results have shown that: (i) samples of synthetic FPN can be easily drawn by using our model; (ii) the synthetic FPN rendered by our abstraction is objectively and subjectively a proper approximation to the actual FPN observed in infrared FPAs. Also, we have presented two interesting applications of our model: (i) striping noise in hyperspectral cameras can be regarded as a special case of our model, and (ii) a previously reported NUC method has been enhanced by simply employing our model for FPN.

\section{References}

1. Dereniak, E.L., Boreman, G.D.: Infrared Detectors and Systems, 1st edn. John Wiley \& Sons, Inc. (1996)

2. Holst, G.C.: CCD Arrays, cameras, and displays. In: SPIE - International Society for Optical Engineering (1998)

3. López-Alonso, J.M., et al.: Principal-component characterization of noise for infrared images. Applied Optics 41, 320-331 (2002)

4. Zhou, H., Liu, S., Lai, R., Wang, D., Cheng, Y.: Solution for the nonuniformity correction of infrared focal plane arrays. Applied Optics 44, 2928-2932 (2005)

5. Narayanan, B., Hardie, R.C., Muse, R.A.: Scene-based nonuniformity correction technique that exploits knowledge of the focal-plane array readout architecture. App. Optics 44, 17 (2005)

6. Torres, S.N., Hayat, M.M.: Kalman filtering for adaptive nonuniformity correction in infrared focal plane arrays. JOSA-A Opt. Soc. America 20(5) (2003)

7. Qian, W., Chen, Q., Gu, G.: Space low-pass and temporal high-pass nonuniformity correction algorithm. Optical Review 17, 24-29 (2010)

8. Hardie, R.C., Douglas, R.D.: A map estimator for simultaneous superresolution and detector nonuniformity correction. EURASIP J. Advances Signal Proc. (2007)

9. El Gamal, A., Fowler, F., Min, H., Liu, X.: Modeling and estimation of FPN components in CMOS image sensors. In: Proc. SPIE, vol. 3301, pp. 168-177 (1998)

10. Godoy, S., Pezoa, J.E., Torres, S.N.: Noise-cancellation based nonuniformity correction algorithm for infrared focal-plane arrays. Applied Optics 47 (2008) 
11. Gómez-Chova, L., Alonso, L., Guanter, L., Camps-Valls, G., Calpe, J., Moreno, J.: Correction of systematic spatial noise in push-broom hyperspectral sensors: application to chris/proba images. App. Optics 47(28) (2008)

12. Leathers, R., Downes, T., Priest, R.: Scene-based nonuniformity corrections for optical and SWIR pushbroom sensors. Optics Express 13(13), 5136-5150 (2005)

13. Fischer, A.D., Thomas, T.J., Leathers, R.A., Downes, T.V.: Stable scene-based non-uniformity correction coefficients for hyperspectral SWIR sensors. In: IEEE Aerospace Conference, 2007, pp. 1-14 (2007) 